\title{
Conectados, demasiado conectados. Poder, técnica y virtualización desde el pensamiento de Paul Virilio
}

Marco Mallamaci ${ }^{1}$

Recibido: 13 de junio de 2018 / Aceptado: 18 de octubre de 2019

Resumen. Este trabajo problematiza el poder y lo político en la era digital desde las ideas de Paul Virilio sobre las sociedades cibernéticas y la red virtual de hiperconectividad. Tras una introducción contextual sobre Virilio, se desarrollan los conceptos de tiempo, velocidad, poder y cronopolítica. Luego, se estudia la categoría de cuerpo político y la crítica de Virilio sobre las revoluciones técnicas; finalmente, se sintetizan las relaciones entre el poder, lo político y la digitalidad del siglo XXI.

Palabras claves: poder; técnica; comunicación; medios; cronopolítica; velocidad.

\section{[en] Connected, all too connected. Power, technique and virtualisation based on Paul Virilio's thought}

\begin{abstract}
This paper problematises the power and the political dimension in the digital era, based on Paul Virilio's thought, which proposes a study on cybernetic society and the virtual web. After a contextual introduction on Virilio, the text develops the concepts of time, speed, power and chronopolitics. Then the idea of political body and the Virilio's critical perspective on technical revolutions are studied. Finally, some relations between power and politics on the digital technical horizon of the $21^{\text {st }}$ century are summarised.
\end{abstract}

Keywords: power; technique; communication; media; chrono-politics; speed.

Sumario: 1. Introducción; 2. Tiempo, velocidad y poder; 3. Los tres cuerpos de lo político; 4. Una crítica a la tecnocracia; 5. Conectados, demasiado conectados; 6 . Referencias bibliográficas.

Cómo citar: Mallamaci, M. (2020): “Conectados, demasiado conectados. Poder, técnica y virtualización desde el pensamiento de Paul Virilio”, en Revista de Filosofia 45 (2), 247-265. 


\section{Introducción}

Si la imprenta y la máquina a vapor transformaron las dinámicas sociales a escala mundial, el paso del siglo XX al XXI ha sido testigo de una mutación aún más decisiva para la condición humana, lo político, lo histórico, lo económico y lo cultural. Las pautas del poder han sido reconfiguradas por una nueva matriz de generación de conocimientos, comunicación, producción y procesamiento de información. Se trata de algo equiparable a la constitución de las sociedades industriales, en el sentido de que, más allá del avance técnico de las máquinas de producción y la electricidad, se conformó un proceso ubicuo de convergencia para la cultura a nivel mundial: la ciencia, la política, la guerra, la producción, la economía, la educación, las ciudades, etc. fueron atravesadas por la industrialización. Luego, con el paso del modelo fabril hacia las sociedades postindustriales de la hiperconectividad digital, se cruza un umbral donde el capitalismo se trenza sobre la tecnología, la informática, la digitalización, las máquinas y el desarrollo de la inteligencia artificial. El eje está en la ecuación "humanidad-máquinas-digitalización-virtualización" y en los modos de conceptualizar los vínculos con la técnica. Se trata de dinámicas que trabajan sobre los hilos culturales; abordar dicha textura sociohistórica tiene que ver con pensar los nuevos modos del poder que allí se conforman.

Luego de Foucault ${ }^{2}$, el poder no solo pudo ser definido como una práctica que circula entre los sujetos de manera difusa, sino que tomó cuerpo una serie de categorías que lo conceptualizan desde una terminología relacionada con lo técnico: técnicas de gubernamentalidad, tecnologías de poder, dispositivos, tecnologías del yo, etc. Pero dicho gesto conceptual no implicó (en Foucault) el ingreso en la trama específica de las rupturas tecnológicas ${ }^{3}$ que experimentaron las sociedades durante el siglo XX: la teletransmisión, la comunicación radial, la televisión, el computo de datos, las redes virtuales de conexión, etc. Si bien su análisis enfoca lo discursivo, lo jurídico, los modos de resistencia y la espacialidad; su idea de tecnologías de poder no implica la profundización de la dimensión técnica contemporánea, la medialidad, las telecomunicaciones y la informática como tramas del poder (Foucault 1989; 1990; 2009). Tal vez, cuando Foucault conceptualizó la sociedad disciplinar y el biopoder, dicha configuración era lo que el mundo ya no era. Hacia mediados del siglo XX la biopolítica ya se había trenzado con ciertas dinámicas postdisciplinares y modos de interacción que emergían de las tecnologías de teletransmisión, del procesamiento de datos y de la programación.

Tal como lo plantea el economista Rifkin ${ }^{4}$ (2011), las problemáticas del poder y lo social en el siglo XXI solo tienen un camino explorable: la tecnología. Ya no se puede pensar el poder sin lo técnico. Hasta la Modernidad la técnica había sido pensada sobre una discontinuidad que marcaba el límite entre lo humano y lo maquínico, entre la dimensión orgánica de la vida y las funciones artificiales de los

2 Se toma como punto de partida la perspectiva foucaultiana del poder (Foucault 2000, pp. 25-32), no para ingresar en su estudio detallado, sino como referencia y punto de partida para exponer la formación de categorías que desplazan el problema del poder hacia la dimensión tecnológica de las telecomunicaciones, la electrónica, la informática, etc. Para ampliar el concepto de poder consultar Castro (2010).

3 Consultar la obra de Kittler $(1995 ; 1999)$ para profundizar la relación entre la arqueología foucaulteana y la técnica como a priori histórico.

4 Le referencia a Rifkin es para ubicar el rol central de la tecnología en la trama política del siglo XXI, se pueden consultar las críticas específicas a la perspectiva tecnocrática que defiende Rifkin en (Sadin 2018). 
objetos técnicos. Con la Revolución Industrial se da un quiebre donde lo artificial se trenza con lo sociopolítico, mientras comienza a desmembrarse la evidencia de que la dureza de la máquina es lo opuesto a la organicidad de lo biológico. La conquista tecnocrática del planeta marcó el horizonte de las sociedades globales y el ingreso a una nueva trama del poder donde los conceptos modernos de soberanía, democracia, libertad, etc. comienzan a desarticularse. Dicho horizonte demanda categorías específicas, mientras a lo largo de la segunda mitad del siglo XX la biopolítica dio lugar a un amplio trabajo analítico sobre el liberalismo y sus dispositivos; las transformaciones de las sociedades globales y las articulaciones entre la tecnología, la comunicación, el mercado, el consumo y la producción, han conformado un tejido funcional que excede las posibilidades teóricas de dicho biopoder. Con la conjunción de los transistores, las placas de silicio y la red virtual del ciberespacio, se forma un complejo que despliega nuevas lógicas de subjetivación, de gubernamentalidad, de formas de intercambio y de circulación. Un autor que propone un esquema donde se interrelaciona dicha serie de elementos es Paul Virilio.

La obra de Virilio entreteje lo social, el poder, lo político y la historia sobre los ejes de las transformaciones técnicas, el espacio, el tiempo, la velocidad, la urbanidad, las formas de comunicación, la guerra, etc. El vector fundamental es la técnica como un elemento relacionado con el tiempo, desde donde el fenómeno de la velocidad es el eje para comprender las transformaciones sociales. De allí surgen categorías como las de cronopolítica, dromología, tiempo absoluto, etc. desde las cuales se puede atravesar la trama escurridiza del capitalismo en la era digital.

\section{Tiempo, velocidad y poder}

Virilio es un autor que hipnotiza con la creatividad de aquellos que han vivido en los bordes de las clasificaciones disciplinares; su obra cruza lo filosófico con la historia, lo fenomenológico, categorías físicas, enfoques sobre la comunicación y lo mediático, análisis de lo bélico, la urbanidad y las ciudades. El mismo Virilio se autodefine como un urbanista mientras construye un sistema conceptual donde despliega una crítica de la técnica y del progreso.

Sin [...] libertad para criticar la técnica, tampoco hay progreso técnico, sino un condicionamiento solamente [...], cuando este condicionamiento es cibernético, como [...] con las nuevas tecnologías, la amenaza es considerable. [...] estamos a finales del $[\ldots] \mathrm{XX}$, y el debate $[\ldots]$ no parece tener en cuenta todo lo que hemos vivido $[\ldots]$ con el progreso. En el XIX podía existir cierta ingenuidad ante el progreso técnico [...]. Las [...] tecnologías de la cibernética [...]; la puesta en red de las relaciones y de la información $[\ldots]$ son $[\ldots]$ portadoras de la perspectiva de una humanidad unida, aunque al mismo tiempo [...] reducida a una uniformidad. (Virilio $1997^{\mathrm{a}}$, pp- 13-14)

La crítica de Virilio en referencia al poder y la técnica tiene su eje en el concepto de velocidad. Su análisis de las transformaciones de los medios de transporte, la revolución de las transmisiones, los cuerpos políticos, el ciberespacio, los accidentes técnicos, etc. surgen del cruce entre el espacio y el tiempo, de donde resulta el movimiento y la velocidad. Esto implica que las transformaciones históricas y sus efectos culturales pueden ser explicados desde la aceleración del tiempo mundial 
y los diversos modos de concebir los territorios, las relaciones, las conexiones y el movimiento de los cuerpos. La noción de velocidad guía toda la obra de Virilio, el eje está en su formación fenomenológica en torno a la obra de Merleau-Ponty. Se puede plantear que la velocidad no es precisamente un fenómeno, sino una especie de relación entre fenómenos. Si para Merleau-Ponty tanto la percepción como la intencionalidad implican la corporalidad y el mundo, para Virilio la velocidad es una condición en relación a la aparición sensible-fenoménica de la realidad.

No olvidemos nunca, en efecto, que la verdad de los fenómenos siempre está limitada por su velocidad de surgimiento (Virilio 1997b, p. 39.)

En Virilio la velocidad es el primer modo de mediatización de la experiencia y de la realidad. La fenomenología parte de las formas del espacio y el tiempo; la velocidad es una variable de relación entre espacio y tiempo, de modo que es relatividad: no tanto un fenómeno, sino una relación entre fenómenos. Este tipo de planteos ha sido criticado por tratarse de un cruce entre lo filosófico y las categorías físicas de Einstein y Newton. Para este último el espacio y el tiempo, en sentido absoluto, eran estructuras vacías, estáticas y homogéneas; luego de Einstein se transformaron en relatividades enlazadas a la aceleración y el valor absoluto de la luz. Con lo cual, la velocidad no sería estrictamente un hecho fáctico, sino una relación fenoménica. Lo llamativo de la idea de velocidad en Virilio es el cruce con la dimensión política, lo urbano, lo territorial y el poder. Si la velocidad es una relación entre fenómenos y puede ser pensada como un medio, cabe pensar en términos de un medio de control, de comunicación, de visión y de percepción. La velocidad, lo trayectivo, el topos, el cuerpo, la técnica y la medialidad se entrelazan para plantear una dromología y una cronopolítica que permiten esbozar el horizonte problemático de la humanidad cibernética del siglo XXI. Mientras el biopoder disciplinar fue el modelo que explicó las pautas del poder en el capitalismo avanzado, la sociedadred del globo digitalizado impone un escenario postdisciplinario que exige nuevas categorías de análisis.

Si la fenomenología piensa el tiempo y el espacio como formas de las intuiciones, la ecuación se completa con el movimiento; allí, la velocidad es una relación entre tiempo y espacio. Virilio desarrolla esta ecuación desde las ideas de "vector" y "trayecto"; se trata del vehículo y el movimiento (desplazamiento), respectivamente. La construcción de este esquema deriva en un interés específico sobre lo "trayectivo"; si se conjugan el espacio, el tiempo, el vector y la velocidad, se obtienen trayectos. Desde lo "trayectivo" se abre una fenomenología que se traduce en tipos de proximidad: la "proximidad inmediata" (el ágora, el foro o los pasajes de las urbes); la "proximidad metabólica" (movimiento animal); la "proximidad mecánica" (revolución de los transportes); y la "proximidad electromagnética" (revolución de las transmisiones). La propuesta de Virilio es pensar la complejidad social, histórica, política, económica y cultural en torno al trayecto y el ser del movimiento.

Sobre lo trayectivo se forma el principio de inteligibilidad de la velocidad. ¿Cómo se podrían relacionar el poder, la democracia, las instituciones, las formas de gobierno y la circulación económica con lo trayectivo y la velocidad? Si el vector es el vehículo del fenómeno en movimiento (trayecto), el grado de aceleración que dicho vector imprime a un trayecto particular, determina un tipo específico de velocidad: velocidad mínima, velocidad relativa, velocidad de liberación y velocidad absoluta. 
Cada uno de dichos modos de velocidad no es otra cosa que una relación particular entre el espacio y el tiempo. Para Virilio dichos tipos de celeridades consisten en modos de relaciones entre el espacio, lo extensivo y lo material, respecto al tiempo, lo intensivo y lo inmaterial. De estas conjunciones se derivan los caracteres "relativo" y "absoluto" de la velocidad. Si se habla de velocidad es imposible no referir toda la serie de elementos al concepto de "aceleración"; lo cual se puede entender como el movimiento que inclina la relación entre el espacio y el tiempo hacia este último; por el contrario, la desaceleración consiste en un movimiento inverso. Acelerar es acentuar el tiempo, lo intensivo y lo inmaterial, mientras desacelerar es remontar la relación hacia la extensión del espacio y su gravidez material. Esto deriva en que toda forma de aceleración tiende a lo inmaterial y al predominio del tiempo sobre el espacio. ¿Por qué este planteo, más cercano a la teoría física que al análisis del poder, puede ser referido a lo político o a lo histórico?

Cuando Virilio se declara como un urbanista y no como un filósofo, pone en foco a la ciudad como la mayor forma política de la historia; la ciudad es el lugar de los trayectos. En la urbe surge el lugar de la proximidad entre los humanos; al existir la ciudadanía, existe la organización del contacto y de los trayectos entre grupos.

Cuando se dice que la ciudadanía está unida a la tierra $[\ldots]$ se olvida $[\ldots]$ el trayecto, [...] la naturaleza de la proximidad que une a los seres humanos entre ellos en la ciudad. Proximidad inmediata con el ágora, el foro y el atrio; proximidad metabólica con el caballo; proximidad mecánica con el tren y la revolución de los transportes; $\mathrm{y}$, finalmente, proximidad electromagnética con la globalización [...] (Virilio 1997ª p. 42)

Para Virilio la historia ha sido una urbanización del espacio: los burgos, las ciudades, las metrópolis, etc. Se trata de pensar la forma-ciudad. El desafío pasa por conjugar una morfología urbana con las categorías de velocidad, trayecto, movimiento, proximidad y vector. Aquí comienza a esbozarse la idea de una dromología y una cronopolítica.

Virilio distingue tres clases de velocidades: "relativa", "de liberación" y "absoluta". Las clases de aceleraciones determinan las grandes revoluciones dromológicas. La velocidad relativa caracteriza la revolución de los transportes, mientras que la velocidad absoluta define la revolución de las transmisiones. En cuanto a la velocidad de liberación, señala un grado particular de intensificación de la velocidad relativa, una especie de liberación de la aceleración, correspondiente al momento de transición que lleva de una a otra etapa. La velocidad relativa supone la primera crisis profunda del continuum espaciotemporal sobre el que se desarrolla la humanidad, con ella la duración comienza a imponerse a la extensión y el movimiento de los vehículos comienza a prevalecer sobre el espacio recorrido. Previo a la revolución moderna de los motores automáticos y la primera aceleración cultural impulsada por los transportes a combustión, el tipo de proximidad que dominaba la dimensión humana era la proximidad inmediata de la aldea o el burgo y la proximidad metabólica de lo animal, mientras que a partir de los transportes motorizados de la Modernidad surge la proximidad mecánica; o sea un nuevo tipo de trayecto. El automóvil, el barco o el tren marcan la importancia que cobra lo trayectivo, el recorrido y la circulación por sobre el espacio y la extensión.

La velocidad que producen los vehículos metabólicos o mecánicos es relativa en el sentido de que la aceleración, que siempre supone una primacía de la duración 
sobre la extensión, no prescinde del espacio físico. Se trata de una ganancia temporal por sobre lo espacial, pero siempre sobre la propia relatividad entre lo intensivo y lo extensivo. La velocidad relativa está fundada en un tiempo lineal, secuencial y cronológico; o sea, las pautas temporales e históricas que dominan todo el pensamiento moderno. Dicho tiempo lineal de la Modernidad sustituye la temporalidad cíclica de la Antigüedad (comprendida en función del movimiento de los astros) y supone la primera liberación importante del tiempo con respecto al movimiento. El tiempo (en sentido moderno) regular, medible y controlable, con la aceleración, deja de ser concebido en función del movimiento; por el contrario, pasa a ser el movimiento el que es concebido en función del tiempo. De este modo, la ecuación entre el movimiento y el desplazamiento, al depender directamente del tiempo cronológico, se vuelve lineal y progresiva, como el de un ferrocarril o el de un automóvil. El tiempo cronológico es un tiempo pensado según la instancia del presente: el pasado se concibe como lo que fue presente y el futuro como lo que será presente. Dicho tiempo lineal hace del espacio una extensión homogénea. Para la episteme moderna eurocentrada, el tiempo y la trayectoria cronológica progresan según un futuro que adviene y un pasado que se conserva como tradición.

En Virilio la aceleración de la Modernidad y la cultura del progreso culmina en la velocidad absoluta, lo cual tiene que ver con una especie de escalada dromológica desencadenada con la revolución de los transportes. El horizonte de la velocidad absoluta impone una situación compleja que abre el ingreso hacia un nuevo tipo de sociedad, hacia sistemas de formaciones sociales ligados a los fenómenos de comunicación y al elemento de la información. Si en las sociedades antiguas el tiempo se concebía cíclicamente y en las modernas se imponía una linealidad cronológica que avanza desde un pasado que fue presente hacia un futuro que será presente, el horizonte dromológico del siglo XXI y la velocidad absoluta parecieran traer un mundo donde el presente ya no pasará más (Virilio 1997ª, pp. 80-86). Virilio enfoca este punto e insiste en dicho planteo (tal vez enigmático) para plantear lo que podría ser una "cultura de la paradoja". Con la velocidad absoluta, la cultura técnica celebra la verdad incómoda de la cultura paradojal, en la cual se hacen presentes modos trayectivos de "trayectos sin trayectoria". Si la velocidad y el movimiento es una variable relativa entre el tiempo y el espacio, y se acepta que cada transformación técnica que impone un aumento de aceleración inclina la balanza hacia la dimensión temporal (o inmaterial); con la inmediatez de las teletransmisiones y la circulación ciberespacial, el espacio extensivo termina por borrarse en favor de la inmediatez. Entonces, no es que lo trayectivo desaparezca, sino que se transforma en un presente absoluto, donde la velocidad no deja ningún tipo de trayectoria extensiva.

Con el ingreso en la era de la velocidad absoluta comienza a borrarse la oposición entre naturaleza y artificio, o entre humanidad y tecnología: lo tecnológico y lo maquinal se integran en un ambiente continuo de creación, inmediatez y transaparencia. Las instancias de mediación se vuelven totalmente maquinales y tanto las proximidades inmediatas de las antiguas urbes, como la proximidad metabólica de lo animal y la mecánica de los transportes modernos, quedan solapadas bajo la proximidad electrónica del espacio virtual. La aceleración de las transmisiones eléctricas suplanta el binomio moderno "realidad/representación" por un pliegue que hace coexistir paradojalmente lo que (hasta entonces) funcionaba sobre las oposiciones y las linealidades cronológicas: entonces surge una hiperrealidad que 
es, para Virilio, una "estereorealidad"5. La realidad hipermoderna es una realidad trans-aparente. Los acontecimientos ya no se representan, ya no hay tiempo para ello: en cambio, se producen, se presentan, se exponen al ritmo vertiginoso de la simultaneidad y la desaparición. Con lo cual el tiempo paradojal de los trayectos sin trayectos genera un escenario donde no hay cosa más vieja que una noticia de hace una semana. Si bien la revolución de las transmisiones es inseparable de las pautas culturales de la sociedad industrial, al mismo tiempo se trata del fin de dicho modelo y el ingreso progresivo en una sociedad postindustrial. Por eso se puede decir que, cuando Foucault pone de relieve el paso de la sociedad disciplinar al biopoder estaba haciendo visible (en cierto sentido) lo que la sociedad ya no era; el trayecto final del siglo XX ya estaba inmerso en un modelo postindustrial y postdisciplinar. La revolución de las transmisiones moldea una sociedad en la cual la circulación veloz de bienes e información acompaña la "desmaterialización" de la realidad: desmaterialización del poder, de los vínculos, de las representaciones, etc. Esto tiene que ver con que, para Virilio, el progreso dromológico conduce a la abstracción: al aumentar la aceleración también aumenta la supremacía de la inmaterialidad y del movimiento del tiempo sobre la inmovilidad y la materialidad del espacio. Esto deriva en la automatización de la velocidad.

Entonces, con el advenimiento de una sociedad postindustrial, la geopolítica comienza a ser sustituida por una cronopolítica, mientras que la sociedad disciplinaria (analizada por Foucault) comienza a ser reemplazada por la sociedad de control imaginada por Burroughs. La propuesta conceptual de Virilio toma cuerpo en la construcción de dos ejes conceptuales que definen una perspectiva específica: la dromología y la cronopolítica. Poner el acento en la relatividad de los fenómenos en términos de una ecuación entre tiempo y espacio, que se resuelve en la velocidad y el trayecto, lleva a la posibilidad de pensar la textura sociopolítica y el poder en referencia al movimiento. Entonces, para Virilio "toda sociedad es una sociedad de carreras" (Virilio 1997a , p. 17). El centro de este planteo es que cada sociedad extrae su poder de su relación especifica con la velocidad: el ejercicio del poder se corresponde, primordialmente, con el ejercicio de la velocidad. El poder puede ser pensado como control o dominio sobre un territorio, sobre la población o sobre las relaciones, se realiza a partir de la velocidad del movimiento. Esto se traduce en que el poder es "poder dromocrático"; o sea, la velocidad es el poder mismo.

El poder es inseparable de la riqueza y la riqueza es inseparable de la velocidad [...]. Sea en las sociedades antiguas a través del papel de la caballería $[\ldots]$, sea $[\ldots]$ a través de la conquista de los mares, el poder es siempre el poder de controlar un territorio mediante mensajeros, medios de transporte y de transmisión [...](Virilio 1997a , p. 17)

La dromología plantea que el ejercicio del poder se da en términos del control de

\footnotetext{
Para Virilio, luego de la llegada a la luna la humanidad forma una nueva consciencia del tiempo astronómico, único y global, desencadenado por la velocidad de las transmisiones: un tiempo-luz. Esto deriva en la velocidad del live televisivo y el ciberespacio, denominada "estereorealidad" (Virilio 2003a, p. 72).

$6 \quad$ Si bien en la obra de Foucault (1989) se debe diferenciar el poder disciplinario (una anatomo-política del cuerpo humano, que tienen como objeto el cuerpo individual) del biopoder que, a partir del siglo XVIII, trabaja sobre la categoría de población; dicha diferenciación es secundaria para el sentido de este trabajo. El biopoder (Foucault, 2010) y la sociedad disciplinar pueden ser vistas aquí como un proceso histórico en el cual la dimensión biopolítica se configura completando dichos dispositivos disciplinarios (no negándolos) (Revel 2009, p. 86), (Han 2014 ; ; 2014b; 2015). En los trabajos foucaulteanos se puede encontrar el análisis de los mecanismos que permiten pensar en dispositivos que se entrelazan formando un "biopoder disciplinario" (Foucault 2000, p. 230).
} 
un territorio a través de formas de velocidad que actúan sobre el espacio y el tiempo. La velocidad es una dimensión decisiva del ordenamiento de las ciudades, de la actividad política, económica y cultural ${ }^{7}$. La palabra dromología se construye desde el griego dromos, que significa carrera. Sería una disciplina que estudia la velocidad desde el punto de vista de la economía y la política desde la aceleración; con lo cual surge una "política de la velocidad". Conceptualizar el poder desde este enfoque es prestar atención a los modos de aceleración espaciotemporales experimentados por una sociedad ${ }^{8}$. Se trata del estudio del "progreso dromológico", la economía política de la velocidad completaría la economía tradicional en tanto estudio de la acumulación y la circulación de la riqueza. Virilio argumenta que, con la aceleración moderna de la historia, que desemboca en la conquista del espacio a través de la intensificación de la duración del tiempo, las sociedades se vuelven dromocráticas. Hacia el fin del siglo XX, las pautas de poder imponen una equivalencia absoluta entre tiempo y capital. Si el capitalismo se trató primero del valor de uso y luego del valor de cambio, este se habría transformado en una porción medible de tiempo: hoy efectivamente el tiempo es dinero; lo cual ya estaba planteado desde Benjamin Franklin en el siglo XVIII (Weber 2010, pp. 67-98). El capitalismo utiliza el tiempo como medida de trabajo, de la producción, del capital y de sus modos de circulación; para Virilio, esto se debe analizar de la mano de las mutaciones técnicas en relación a la serie espacio-tiempo-sociedad. Primero fue la aceleración de los transportes marítimos, luego los motores y hoy, la aceleración de las informaciones; entonces, se impone una política de la velocidad y del tiempo. La dromología y la cronopolítica encuentran su espacio analítico fundamental en la ciudad; la preocupación de fondo de dicha dromología son los modos de la urbanidad. Para Virilio la velocidad y la ciudad no pueden separarse, se determinan mutuamente según las formas de circulación y la organización de los trayectos ${ }^{9}$. Urbanizar es desarrollar dispositivos que determinan la velocidad de la sociedad y los parámetros del tiempo y el espacio en sentido político. Los ciudadanos son aquellos que trazan las trayectorias urbanas, son seres de circulación y velocidad.

Si la analítica del biopoder logró conceptualizar los dispositivos disciplinarios de la sociedad, pero no puso de relieve el fenómeno de la técnica en sí mismo, el fin del milenio trajo una textura epistémica que permite pensar el poder desde

Consultar los estudios de Innis (1950) sobre los medios en relación al poder y las referencias de Kittler (1995, 1999) a Innis y McLuhan.

8 La relación entre el tiempo y la dimensión sociopolítica se puede encontrar en diversos trabajos donde se ponen de relieve los patrones temporales en términos de construcción social. Desde dichos enfoques el tiempo puede ser pensado como una cuestión política donde se construyen complejas relaciones estructurales que configuran pautas sociales y de poder en referencia a las condiciones técnicas. Se puede ampliar la temática consultando la relación entre las sociedades modernas y la temporalidad en los trabajos de Anthony Giddens y la categoría de "vaciado temporal" (1993, pp. 28-32); en Harmut Rosa (2016), quien estudia cómo las estructuras temporales modernas se transforman gobernadas por las reglas y las lógicas de procesos de aceleración; o bien en planteos como el de Jonathan Crary (2015), donde se hace referencia a la durabilidad de lo social en un sistema económico global que trabaja a tiempo completo. Cabe aquí sumar el trabajo de Judy Wajcman (2017), quien estudia la relación entre sociedad y alta aceleración, particularmente en torno al capitalismo digital. Otra referencia fundamental se puede encontrar en la obra de Bernard Stiegler, quien, a partir de Heidegger, Simondon y Derrida, elabora una filosofía de la técnica donde el tiempo funciona como eje de problematización. Allí Stiegler (2002) hace algunas breves referencias al pensamiento de Paul Virilio y sostiene que la relación del tiempo y el espacio solo sería pensable a partir de la velocidad, dimensión que (según el autor) en aquel contexto seguía siendo impensada.

9 Consultar la conceptualización de la ciudad como un modelo de comunicación en la Filosofía de los Medios de Kittler (2014, pp. 181-213). 
otra complejidad. Esto no anula las aristas desarrolladas por Foucault, sino que penetra el tejido histórico poniendo de relieve algo que suele ser denominado como postdisciplinario, pero que desde la óptica de Virilio toma la forma de una dromocracia o un cronopoder.

\section{Los tres cuerpos de lo político}

Cuando Virilio problematiza la técnica no solo piensa en lo eléctrico, la mecanización, la productividad o los transportes, sino que propone un elemento esencial en relación al lugar. Dicha relación cobra sentido en la idea de cuerpo: el cuerpo territorial, el cuerpo social y el cuerpo biológico. La historia de la humanidad muestra que las tecnologías han producido diversos tipos de velocidades modificando paulatinamente cada uno de dichos cuerpos. Esto tiene que ver con que, en última instancia, la técnica se trata de una dimensión que busca conquistar el espacio a partir del tiempo, o sea de atravesar y reorganizar la materialidad del cuerpo a partir de la aceleración inmaterial del tiempo. Virilio avanza en su razonamiento proponiendo que, el lugar de las mutaciones técnica termina derivando en la dimensión intraestructural. Se trata una nueva circunstancia del progreso dromológico, cada nuevo capítulo de dicho progreso de aceleración profundiza la acción de la velocidad sobre el cuerpo territorial, sobre el cuerpo social y finalmente sobre el cuerpo biológico. Respectivamente, se trata de la infraestructura, la superestructura y la intraestructura; aquí, lo central está en los modos de aceleración y velocidad que fabrican las innovaciones técnicas.

De la relación entre los tres cuerpos y el progreso dromológico se desprenden funcionalidades económicas y políticas. La velocidad depende fundamentalmente del cuerpo, este se trata del plano primordial sobre el que se experimenta la vida. Los tres tipos de cuerpos están ligados entre sí y pueden ser considerados según un orden de implicancias. El cuerpo territorial es el propio planeta Tierra, tiene que ver con un enfoque geográfico. Allí se enlazan el clima, el suelo, la división del medio, los aspectos físicos del terreno, el registro elemental del espacio urbano o rural, los modos de organización del suelo, del medio natural. El concepto de territorio puede ser entendido como un producto de la emancipación del terreno primordial por la acción de la velocidad, la posibilidad de controlar un territorio se da por la velocidad que actúa sobre el espacio. Se trata del dominio del territorio por medio de la comunicación, los mensajes, el transporte, las conexiones, las transmisiones, etc. Un medio se caracteriza por la relación entre el terreno y el territorio; lo que Virilio llama un cuerpo territorial: siempre es un problema de velocidad.

En cuanto a las distintas clases de proximidades que se forman en la dimensión del cuerpo social, son definidas por las relaciones que los cuerpos y los objetos establecen en un medio territorial, relaciones que a su vez dependen del tipo de aceleración que las configura: transportes terrestres, marítimos, aéreos, medios electrónicos, teléfono, radio, televisión, etc. Al ingresar en la dimensión del cuerpo social surge todo lo relacionado con la sociedad en tanto instancia de intercambio, vínculo e interacción; o sea modalidades de organización de la circulación de personas y bienes. Si el cuerpo territorial provee el horizonte tecno-geográfico, el cuerpo social dispone un horizonte complejo, a partir del cual se conforma el imaginario colectivo; mientras que la dimensión del cuerpo biológico permite la vivencia singular del mundo a través de un horizonte individual. El progreso dromológico de la técnica 
se trata de la conquista, primero del cuerpo territorial, luego del cuerpo social y finalmente, desde el último tercio del siglo XX, la conquista del cuerpo biológico. Si a mediados del siglo XX la técnica y lo mediático fueron pensados como una dimensión protésica, la tecnociencia contemporánea conduce a una miniaturización del objeto técnico que permite su ingreso en el propio cuerpo animal. El tercer tipo de cuerpo (animal) se corresponde directamente con la vida biológica; mientras el segundo permite la producción, el tercero posibilita la procreación; al mismo tiempo, el primero (territorial) da el punto de apoyo y las condiciones para ambos (suelo, clima, estabilidad).

Cada cuerpo genera un horizonte de movimiento. Sobre el cuerpo territorial se destaca toda la escena de la vida humana, el cuerpo social genera la textura de los imaginarios colectivos, donde se tejen las tramas de interacción y el cuerpo biológico se desarrolla en la vivencia singular del mundo, o sea un horizonte "individual". Los tres tipos de cuerpos (territorial, social, biológico) trabajan enlazados a la técnica, al movimiento, al trayecto y a la velocidad. Así como el movimiento implica un trayecto y la velocidad implica el movimiento, toda la ecuación implica la noción de cuerpo. El tejido entre lo territorial, lo social y lo biológico toma forma en una especie de ritmología, donde surge una economía política de la velocidad. Si bien la idea de un tipo de movimiento y sus modos de aceleración se puede relacionar con la velocidad que posibilita un vehículo, en definitiva, lo axial está en la velocidad que experimenta la dimensión del cuerpo. Las tres grandes revoluciones de la Modernidad derivan en relaciones específicas que marcan el progreso dromológico según ciertas dinámicas entre la velocidad y los diferentes tipos de cuerpos. Con la revolución de los transportes comienza la gran conquista técnica del espacio físico, o sea, del cuerpo territorial. Durante el siglo XIX el espacio comienza a ser conquistado por la reducción de la duración de los tiempos de desplazamiento; las sociedades modernas logran ganarle tiempo al tiempo mediante un progresivo dominio técnico del espacio por medio de los diversos dispositivos de aceleración de los trayectos. La aceleración de la Modernidad logra conquistar el espacio por medio del tiempo y el resultado es que el planeta se empequeñece.

Al pensar el poder desde una perspectiva dromológica se pone de relieve la modificación del lugar, del espacio, de lo urbano, del territorio y del cuerpo. Cada mutación técnica, desde el uso del transporte a las redes de teletransmisiones afecta la relación entre los cuerpos, la materia y los conceptos tradicionales de topos, territorio y extensión. El progresivo avance del cibermundo enlazado al previo despliegue del teléfono, la radio y la televisión permite modos de aceleración y de trayectos que forman la ecuación en términos de telepresencia. Entonces el cuerpo territorial, el social y el biológico pueden ser deslocalizados; el lugar deja de estar circunscripto al "aquí y ahora". Si la historia de la ciudad y la historia de lo político forman una sola trama enlazada a diversos pedazos de territorio, el aumento exponencial de la velocidad moderna transforma los modos de percibir lo corporal y lo material, abriendo una dinámica que deriva en el desvanecimiento del lugar y del topos tradicional. La telepresencia teje una nueva forma de enramar la extensión a la intensidad, el espacio y el tiempo resuelven su tensión fenoménica en algo que antes era inexistente: la virtualidad y la cibervelocidad del presente constante e inmediato. Entonces el progreso dromológico de la técnica en el siglo XXI dibuja un mapa de trayectos sin territorio y de desplazamientos sin extensión. El tiempo pareciera ganar la carrera auto-devorándose en un cuerpo social cronopolítico. 


\section{Una crítica a la tecnocracia}

La perspectiva fenomenológica con el eje puesto en la velocidad es la llave para que Virilio pueda contar una historia del poder y de las formaciones políticas desde los sistemas técnicos que funcionan como condición de posibilidad para modos de trayectividad y urbanidad. En Virilio se trata de una historia crítica. Cada paso en el progreso tecnológico desencadena un progreso dromológico; por lo tanto, la velocidad es inseparable de la técnica y la dromologia se ocupa de ella.

[...] sin libertad para criticar la técnica, tampoco hay "progreso técnico", sino un condicionamiento solamente. (Virilio 1997a , p. 13)

Para Virilio el concepto de poder tiene uno de sus ejes en el "poder dominar" y la velocidad es el poder primordial. La imagen de todos los líderes de la historia tiene que ver con conducir las energías y el ritmo de la sociedad que controlan. No hay poder sin ley, sin formas de regulación, sin constituciones y sin tipologías de la justicia. La justicia y la organización del poder son dinámicas que controlan la riqueza, la economía y los repartos en general; en última, son modos de ordenar los tipos de trayectos y las velocidades del cuerpo social; el poder trabaja trenzado siempre sobre una ritmología política. En el pasado, las noblezas fueron clases de velocidad, al igual que lo fueron las caballerías. La organización social del poder según los repartos entre campesinados y nobles no es independiente del hecho de que unos solo cuenten con cuadrillas de bovinos y otros con medios de transporte más potentes. Tanto en la Antigüedad, como en la primera Modernidad, la ecuación que resolvía el problema del poder tenía que ver con velocidades relativas, las del caballo, el navío o bien las de vehículos como el tren o el automóvil (en la Modernidad más avanzada). Se trata de trayectos relativos porque las aceleraciones siempre suponen una primacía de la duración sobre la extensión, son tipos dromológicos que no pueden prescindir de la espacialidad física. Dichos tipos de cuerpos sociales, organizados sobre la aceleración relativa pueden poner el acento en la democratización del cuerpo social. En las primeras democracias griegas el poder estaba en el control del transporte marítimo, por eso los atenienses podían elaborar constituciones políticas afirmando que: "Los que gobiernan los navíos deben gobernar la ciudad." (Virilio 1997ª p. 19). La técnica que define el tipo de movimiento trayectivo de una sociedad es inseparable del modo en que esa sociedad entiende el ejercicio del poder; las democracias tienen que ver con tipos de velocidades relativas (por ejemplo, los navíos).

Los tres tipos de velocidad que concibe Virilio definen formas de aceleración que configuran el cuerpo social; las velocidades relativas tienen que ver con la tensión entre el espacio y el tiempo en una dimensión donde la aceleración debe vencer la gravedad de la materia extensa. Con el desarrollo de los motores a combustión se da una revolución de los medios de transporte terrestres y marítimos que deriva en la conquista de dicha velocidad relativa. Con el desarrollo de la aeronavegación y los cohetes espaciales se generan las condiciones para alcanzar la velocidad de liberación de la gravedad terrestre; se trata de una intensificación donde la relación espaciotemporal da un salto y los trayectos pueden ser pensados, por ejemplo, a la velocidad del sonido. Por último, con la revolución de las transmisiones los trayectos pueden viajar en tiempo real, sin peso, a la velocidad de la luz e imponiendo una especie de telepresencia inmediata; es la dimensión de la velocidad absoluta. Aquí el 
movimiento se da en el puro tiempo sin la inscripción y el peso del espacio extensivo. Cada uno de estos niveles de progreso dromológico se corresponden con dispositivos y configuraciones del poder. Las democracias solo pueden surgir en etapas donde la velocidad social trabaja sobre la relatividad del peso extensivo del territorio; cuando los griegos navegaban eran necesarios muchos hombres para remar, la necesidad de ese tipo de trabajo permite pensar el reparto equitativo de las fuerzas; lo cual cambia a partir de que la humanidad conquista la velocidad de la luz. Las transmisiones electromagnéticas y el imperio de la velocidad absoluta plantean un escenario donde la inmediatez y la hipermovilidad sobre el tiempo real abren el interrogante sobre un nuevo tipo de sociedad, un nuevo cuerpo social configurado sobre la telepresencia global y los trayectos acompasados sobre la velocidad de la luz. Para Virilio, lo propio de la velocidad absoluta es ser también un poder que permite un control absoluto; de hecho, las características de las transmisiones eléctricas y la entrada en la cibercomunicación pueden ser predicadas desde los atributos de lo divino: la ubicuidad, la instantaneidad y la inmediatez. Entonces, el siglo XXI debería traer algo que ya no tiene nada que ver con la democracia; los multimedia y su entrada en la velocidad absoluta del ciberespacio nos enfrenta al problema de encontrar un modo de poder coherente con la vieja idea de democracia y a la vez con la velocidad y la aceleración de los trayectos de tiempo real.

A partir del siglo XVIII el planeta fue transformado por la industrialización; para Virilio, lo central de dicho proceso fue la revolución de los transportes. Esta modificó el medio de las sociedades; los ferrocarriles, la máquina de vapor, los navíos y el poderío marítimo, derivaron en nuevos parámetros de percepción espaciotemporal. Desde el enfoque dromológico, a partir de aquel momento se abrió el camino hacia formaciones culturales donde se ponen en práctica modos de velocidad industrial; se trata del paso gradual de la geopolítica a la cronopolítica. Durante el siglo XIX, la construcción de redes ferroviarias que conectaran regiones, centros de intercambio económico, puertos y urbes, fue el eje de las estrategias gubernamentales. Entrar en el ritmo del "progreso moderno" era sinónimo de tejer el territorio con vías, estaciones y locomotoras. Virilio cita a Audibert, quien, cuando se dieron a conocer las vías férreas, decía:

Si logramos hacer llegar los trenes a la hora en punto, habremos dotado a la humanidad del instrumento más eficaz para la construcción del nuevo mundo. (Virilio 1997ª p. 20)

Luego de la revolución de los transportes la idea de la temporalidad, en sentido social, cambió para siempre. La cronopolítica y la carrera del tiempo contra el tiempo es el prólogo para la posterior entrada en la cibernética. La era del poder cronopolítico se caracteriza por una pérdida de afecto por el cuerpo territorial; de hecho, a partir del capitalismo industrial y la Modernidad comienza el fin de la vida humana como campesinado; la oposición campo/ciudad termina torciéndose en favor de la última y el gran drenaje de las poblaciones rurales hacia las ciudades industriales se traduce (con el paso del siglo XX) en una humanidad hipermóvil. Los cuerpos sociales de la era de la revolución de los transportes toman la forma de masas poblacionales que se concentran en las ciudades o alrededor de las grandes empresas, mientras el ritmo de la vida campesina poco a poco desaparece entre el humo de las locomotoras y los motores que marcan las nuevas formas trayectivas de las urbes. En el siglo XIX el ferrocarril era la llave para, primero la reunión de los pueblos 
europeos en una sola ágora y luego la formación de una democracia mundial. La perspectiva era que las redes ferroviarias favorecerían la convivencia y la solidaridad por medio del intercambio de mercancías, ideas, experiencias, relaciones humanas, etc. (Virilio $1997^{\text {a }}$, pp. 20-21). Hacia la década de 1860 surgía una nueva palabra en la terminología política y económica que intentaba comprender dicho tejido social: "capitalismo" 10 . La aparición del término capitalismo significa tanto la puerta para la crítica hacia aquella sociedad liberal, como (al mismo tiempo) el triunfo de un sistema cultural basado en la idea de "desarrollo económico", impulsado por los engranajes de los mercados, por la confianza en que la producción industrial permitiría comprar y vender barato, masivamente y generar riqueza; se trata de un mundo de abundancia (Hobsbawm 2010, pp. 13-15). Dicho mundo trenzó sus criterios trayectivos sobre las figuras de los Estado-Nación y la red interestatal mundial. Si tradicionalmente se ha analizado cómo la configuración de ese sistema mundial de poder va enlazada a la ciencia, a la tecnología y a la reproducción industrial; Virilio intenta poner de relieve cómo las vértebras de dicho proceso sociohistórico están relacionadas con un progreso dromológico, o sea con una dinámica de aceleración sobre el cuerpo social, sobre el espacio y el tiempo. El triunfo cultural del liberalismo-capitalista y la cultura industrial engranada sobre la mundialización económica son inseparables del ferrocarril, del motor y del empequeñecimiento del planeta, derivado de la aceleración de la revolución de los transportes. Por eso el avance del capitalismo puede traducirse como la entrada en una pauta de poder cronopolítico.

Para Virilio el poder siempre ha sido un fenómeno relacionado con las condiciones de posibilidad para recorrer los espacios y generar trayectos conectivos.

Sea en las sociedades antiguas a través del papel de la caballería (los primeros banqueros romanos eran caballeros), sea en la potencia marítima a través de la conquista de los mares, el poder es siempre el poder de controlar un territorio mediante mensajeros, medios de transporte y de transmisión (Virlio 1997ª p. 17).

El poder en sentido dromológico tiene que ver con cómo las dinámicas del cuerpo social generan la emancipación de lo territorial por la acción de la velocidad; ya sea con los transportes terrestres, marítimos o aéreos. Luego de la revolución de los transportes, aparece otra mutación en el modo de percibir el continuum espaciotemporal; el progreso dromológico avanza hacia la revolución de las transmisiones. A partir de los medios electrónicos de telecomunicación, como el teléfono, la radio y la televisión se conforma el sistema de movilidad en la dimensión de la telepresencia. Esta segunda revolución impulsada a fines del siglo XIX con la prensa masiva y el telégrafo, se intensifica a lo largo de todo el siglo XX empujando las velocidades de propagación. Se avanza entonces hacia una especie de conmoción que sufre la extensión por parte de la temporalidad, la cual se encoge sobre sí misma haciendo de la distancia un problema menor. La conjunción entre los medios modernos de transporte y los medios de transmisión de ondas deriva en que la velocidad lleva a la abstracción de los vectores y de los modos trayectivos.

La crítica de Virilio pasa por preguntar cuáles son los efectos culturales de la aceleración del tiempo mundial. Tal vez el trabajo crítico fundamental pasa por

10 En 1867 se publica El Capital de Marx; luego de 1848 el término ya circulaba y si bien es posible que existiera en las décadas previas, no se han encontrado registros del uso de la palabra "capitalismo" antes de 1849 (Hobsbawm 2010, p. 13). 
comprender en qué sentido pueden enlazarse los nuevos medios de comunicación y transporte como los drones, Internet, lo mutimediático, la domótica, etc. con los antiguos valores que se forman sobre los conceptos de democracia y libertad. Virilio plantea que luego de las revoluciones modernas (transporte y transmisión), el paso del siglo XX al XXI nos enfrenta a la revolución cibernética, allí se eleva una gran fantasía: la democracia virtual. El sueño de que el progreso dromológico entraría en una especie de hiperconectividad democrática e igualitaria, donde la virtualización de los trayectos y el ingreso en el espacio liviano e infinito de la digitalidad permitirían el paso hacia un futuro donde el cuerpo social se abre con más posibilidades para todos y menos exclusión, debe ser enfrentado con la crítica.

Siempre estamos enfrentados a un fenómeno de colaboración o de resistencia. [...] Frente al objeto técnico, $[\ldots]$ hay que volverse crítico. El impresionismo es una crítica de la fotografía y el género documental es una crítica de la propaganda. $[\ldots]$ hace falta $[\ldots]$ una crítica [...] para hacer divergir la relación con la técnica. (Virilio 1997ª pp. 34-35)

La historia de la acumulación de la riqueza y del capital es inseparable de la técnica, de la aceleración y de la transformación de las dinámicas que marcan el ritmo y el funcionamiento del cuerpo territorial, del cuerpo social y del cuerpo biológico. Para Virilio la cara oculta de la capitalización y de la riqueza es la aceleración; primero la de los transportes, luego la de las transmisiones y hoy la de las informaciones. Entonces, se impone una política y una economía de la velocidad, o sea una cronopolítica. El sentido de este enfoque apunta al territorio y a la urbanidad, la ciudad ha sido la forma política fundamental de la historia, la aceleración, la velocidad de la riqueza, su circulación y los modos automáticos de cotizaciones hipermóviles han generado un sistema mediático que puede suplantar lo territorial. Entonces el espesor de la textura urbana tiende a desaparecer en favor del intercambio informático. El siglo XXI impone dicha ecuación desde la dimensión de la velocidad absoluta de la cibernética.

Dicha velocidad absoluta es la culminación del progreso dromológico desencadenado con la revolución de los transportes, es el ingreso a una sociedad ligada a la comunicación, la conectividad y la información. El avance de los transportes y las transmisiones encuentran una velocidad límite, si la aceleración es una relación entre la espacialidad (extensión) y la temporalidad, en favor de esta última, siempre se trata de una tensión con la gravedad terrestre y el trayecto en tanto distancia. Al llegar al dominio de medios como el avión se alcanza la velocidad de liberación de la gravedad terrestre y con ello la supremacía del tiempo de la luz sobre el espacio de la materia. La emancipación del trayecto grávido de la espacialidad terrestre debe ser conjugada con la conquista de la telepresencia por medio de la radio, el teléfono, la imagen instantánea de la fotografía, la imagen en movimiento del cine, la transmisión en vivo, etc. Entonces el horizonte de progreso dromológico está listo para una humanidad que habite la aceleración del ciberespacio. La dimensión de la velocidad absoluta logra reemplazar la superficie del suelo por la superficie lumínica de las pantallas.

El siglo XXI es el siglo de una dromología encarnada en la funcionalidad domótica y en la hiperconectividad cibernética. La antigua perspectiva espacial es reemplazada por la perspectiva en tiempo real del marco de la pantalla y las interfaces hardware-software-humano. La crítica de Virilio busca direccionar toda esta serie 
de elementos hacia el momento en el cual aparece la amenaza de una cibernética social que automatiza la interactividad. Entonces, del mismo modo que existe una economía política de la riqueza y de la acumulación, se impone un pensamiento cronopolítico y dromológico que piense las aristas de una economía y una política de la velocidad.

\section{Conectados, demasiado conectados}

Si cuando Foucault logró conceptualizar la sociedad disciplinar, eso era lo que el mundo ya no era, cuando Virilio propone pensar un poder dromológico y una cronopolítica, tal vez se está frente a lo que ya somos, pero aún no comprendemos. Los cuerpos sociales ya están tejidos por algo que no podemos analizar en sus consecuencias: la conectividad cibernética, la trama trayectiva del espacio virtual y la inmediatez temporal. Es la era de la velocidad absoluta y sus pautas cronopolíticas del poder. El mundo postdisciplinar trae una red de cuerpos sociales que se globalizan sobre una gubernamentalidad algorítmica y un dispositivo de dispositivos que genera modos de subjetivación hiperindividualizados, pero a la vez hiperconectados sobre una dinámica blanda, liviana, fugaz y siempre frágil. De la iluminación de las telecomunicaciones y la aurora del sol artificial emerge un sistema que impone una nueva temporalidad; una especie de tiempo global donde la simultaneidad de la acción prevalece sobre su sentido. El ritmo dromológico de la teletransmisión, primero del sonido y luego de la imagen, construyeron una continuidad audiovisual progresiva que cubre las naciones y atraviesa las fronteras políticas, haciendo mutar el espacio político tradicional en una cronopolítica virtual. Virilio identifica dos aspectos: por un lado, la reducción de las distancias y los nuevos modos de comprender el tiempo, y por el otro, el despliegue generalizado de un enorme dispositivo de telecontrol. Las sociedades telecontroladas explotan la visibilidad de la telepresencia continua, las veinticuatro horas del día, los siete días de la semana, los trecientos sesenta y cinco días del año; se trata de un horizonte de panoptismo que finalmente se traduce en dispositivos algorítmicos de cibercontrol.

Norbert Wiener temía [...] en 1952, que la cibernética, [...] pudiera convertirse en una amenaza para la democracia. [...] se puede llegar a un control total de las poblaciones utilizando la informática y la robótica [...]; la cibernética (del griego kybernana: "dirigir") trata procesos de mando y comunicación entre los hombres y las máquinas. Estas dos poblaciones, la de los seres vivientes y la de los objetos técnicos, pueden $[\ldots]$ entrar en conflicto [...]. (Virilio $1997^{\text {a }}$, pp. 34-35)

Pensar las nuevas pautas del poder desde la perspectiva cronopolítica tiene que ver con intentar conceptualizar las relaciones entre los tipos de velocidades y la realidad virtual del ciberespacio:

La velocidad de escape es la velocidad en la que un cuerpo vence la atracción gravitatoria de otro cuerpo, como $[\ldots]$ una nave espacial cuando abandona la Tierra. La $[\ldots]$ cibercultura, parece cada vez más cerca de [...] esa velocidad de escape" (Virilio 1997b, p. 13) 
El mundo se empequeñece proporcionalmente a su grado de virtualización, las velocidades tienden a ofrecer un tiempo sin espacio, un trayecto sin trayectoria, un instante sin lugar: algo posible de ser llamado virtualidad. La llamada globalización expresa la virtualización de la extensión geofísica. Para Virilio no hay, propiamente globalización, sino virtualización mundializada, o sea un modo de aceleración tecnosocial que alcanza la dimensión del tiempo único: lo que se globaliza es el tiempo.

La problemática abierta a partir de las técnicas telecráticas y la telepresencia tiene que ver con las dinámicas del tiempo real, la velocidad y el tiempo presente. La temporalidad funciona sobre un espesor donde se enlazan el pasado, el presente y el futuro; tejiendo sobre el cuerpo social algo que puede ser llamado tiempo histórico. La telepresencia marca la entrada en algo diverso a dicho tiempo histórico, en una interactividad que deriva en un tiempo único. Entonces se construye un cuerpo social basado en la instantaneidad y la unicidad. Mientras los tiempos de las urbanidades locales registraban la singularidad de la memoria de un lugar, el tiempo único de la cibernética comprime todo el espesor histórico en una trayectividad sin trayecto, en un registro omnipotente que condensa enormes masas de datos (Big Data) sobre la inmaterialidad del espacio virtual. Entonces la interactividad instantánea del siglo XXI se aproxima a la velocidad de la luz. Surge, para Virilio, el tema de la desaparición en la dimensión urbana; la ciudad y lo político en conjunción con la globalidad abren un horizonte de preguntas en torno a la transparencia, la transapariencia y la desaparición en los modos telemáticos del cuerpo social; se trata de la virtualización. ¿Cómo sería un tejido de poder democrático en dicho escenario? ¿O sería la idea de democracia un concepto obsoleto? ¿Se podría pensar en algún nuevo sentido político desde una forma dromocrática bajo una estructura cronopolítica? El holograma del ciberespacio trae consigo un cuerpo social domótico y una nueva urbanidad que se puede conceptualizar con el término de metapolis: lo cual tiene que ver con que mientras más crece la transmisión instantánea de datos e información, más se desvanece el desplazamiento sobre pautas geométricas. Entonces, en el momento en el cual el sistema de telecomunicaciones en tiempo real hace de las superficies puras interfaces, las ciudades se desmaterializan progresivamente; surge el desafío de la urbanización, ya no del espacio, sino del tiempo.

La interfaz audiovisual colma el espacio y la urbanidad política con imágenes, con el vértigo de la velocidad, con la luminosidad, con la acción instantánea y con la certeza de un tiempo que ya no transcurre, sino que se expone. Este es el nuevo horizonte de la velocidad absoluta, la velocidad de las ondas que viajan a través de un medio técnico que aparentemente desmediatiza las relaciones de comunicación en un tejido de hiperconectividad. Con la revolución de las transmisiones se alcanzaba progresivamente el límite de aceleración de los trayectos, el planeta sentía el ingreso en una nueva época y la formación de un nuevo tipo de sociedad, prueba de ello es toda la experiencia de las vanguardias artísticas, la cinematografía, el diseño industrial y el diseño urbano de la primera mitad del siglo XX. Cuando los medios electrónicos se enlazan al problema del cómputo, a la programación, a la informática y a la codificación algorítmica, se consuma el ciberespacio y se cumple lo que Virilio plantea como la "sociedad dromológica"; una sociedad de carreras, un sistema cronopolítico que muta en dromocracia. La sociedad de la comunicación y la información es lo que Virilio denomina sociedad postindustrial; en la dromocracia prima la circulación de mensajes por sobre la acumulación; lo cual termina tomando 
forma funcional por medio de la liviandad etérea y delgada de la luminosidad de las pantallas. Los trayectos del cuerpo social del siglo XXI solo necesitan códigos e interfaces lumínicas.

La crítica de Virilio plantea que cada transformación técnica implica la aparición de un tipo de accidente. Con la velocidad absoluta de los medios electrónicos el tiempo pierde su inscripción trayectiva sobre la extensión material del espacio, se independiza y se deslocaliza. Ingresar en una sociedad que trabaja al ritmo de la velocidad de la luz es una situación que ya no modifica el continuum espaciotiempo, sino que lo accidenta. La virtualidad del espacio desmaterializado hace que la duración se comprima y se abra una espacialidad desconocida. Entonces la temporalidad se quiebra y ya no hay ni antes, ni después, el cuerpo social del siglo XXI vive sobre un constante, inmediato y trans-aparente durante.

A la inversa de la perspectiva del espacio real de la geometría, la perspectiva del tiempo real no está ya limitada por la gravedad terrestre; el horizonte trans-aparente de la pantalla [...] escapa de la gravitación fundándose en la propia velocidad de la luz [...] (Virilio 1997b, p. 49)

La superficie fenoménica ya no marca trayectos lineales, ni circulares, sino que se hunde en la instantaneidad del presente: se trata de un tiempo que ya no se extiende, sino que se expone. La materialidad posee tres dimensiones: la masa, la energía y la información; cada época de la historia y cada sistema de poder político ha privilegiado una de esas dimensiones. Primero fue la masa, la de las murallas, luego fue la energía de la balística y la pólvora, finalmente, la bomba, los misiles y el arma atómica. Hoy se impone una nueva dimensión en base a la información y su velocidad de comunicación instantánea; entonces surge la infowar (guerra informacional); el arma de comunicación masiva es concebida como estratégicamente superior al arma de destrucción masiva. Si es válida la inversión que propone Foucault sobre la definición de Clausewitz y lo político es la continuación de la guerra por otros medios, siendo el poder el tejido escurridizo por el que circula la acción, el siglo XXI abre el juego a una trama que se esconde detrás de un nuevo signo de interrogación: ¿de qué se trata eso que ya somos, pero aun no comprendemos?

The network is robust in its unstructured simplicity. Nodes work all at once with little coordination. They do not need to be identified, $[\ldots]$ messages are not routed to any particular place [...]. Nodes can leave and rejoin the network at will, accepting [...] what happened while they were gone. (Satoshi Nakamoto 2008, p. 8)

$[\ldots]$ ese planeta digital no es ninguna otra cosa, $[\ldots]$ que la sombra informacional integral de nuestra Tierra y sus saberes desde la noche de los tiempos. (Kyrou 2010, citado en Sadin 2017, p. 78)

\section{Referencias bibliográficas}

Abad Dominguez, F. (2006): Filosofía de la comunicación, Caracas, Ministerios de Comunicación e información.

Acevedo, J. (1999): Heidegger y la época técnica, Santiago de Chile, Editorial Universitaria. Augé, M. (2000): Los no lugares, Barcelona, Gedisa. 
Barker, C. (2003): Televisión globalización e identidades culturales, Barcelona, Paidós.

Bell, D. (2007): Cyberculture theorists: Manuel Castells and Donna Haraway, New York, Routledge.

Berardi, F. (2017): Fenomenología del fin. Sensibilidad y mutación colectiva, Buenos Aires, Caja Negra.

Brea, J. L. (2007): Cultura_RAM, Barcelona, Gedisa.

Briggs, A. y Burke P. (2002): De Gutenberg a Internet, Madrid, Taurus.

Castells, M. (2001): La galaxia Internet, Barcelona, Plaza y Janes.

Castells, M. (ed.) (2004): The network society, Massachusetts, Edward Elgar.

Castro, E. (2010): El vocabulario de Michel Foucault, Buenos Aires, Universidad Nacional de Quilmes-Prometeo.

Carpenter, E. y McLuhan, M. (eds.) (1960): Explorations in communication. United States of America: Beacon Press.

Cohen-Seat, G. y Fougeyrollas, P. (1980): La influencia del cine y la televisión, México, FCE.

Crary, J. (2015): 24/7, el capitalismo al asalto del sueño, Barcelona, Editorial Planeta.

Debord, G. (2008): La sociedad del espectáculo, Buenos Aires, La Marca.

Debray, R. (2001): Introducción a la mediología, Barcelona, Paidós.

Derry, T. y Williams, T. (1977): Historia de la tecnología. Tomos I, II y III, Madrid, Siglo XXI.

Echeverría, J. (1999): Los señores del aire: telépolis y el tercer entorno, Barcelona, Destino.

Foerster, V. (1991): Las semillas de la cibernética, Barcelona, Gedisa.

Foucault, M. (2000): Defender la sociedad. Buenos Aires: Fondo de Cultura Económica.

Foucault, M. (2008): El orden del discurso. Buenos Aires: Tusquets.

Foucault, M. (1990a): Historia de la sexualidad. La voluntad de saber. Buenos Aires: Siglo XXI.

Foucault, M. (1980): Microfisica del poder. Madrid: La Piqueta.

Foucault, M. (2010): Nacimiento de la biopolítica. Buenos. Aires: Fondo de Cultura Económica.

Foucault, M. (2009): Seguridad, territorio, población. Buenos Aires: Fondo de Cultura Económica.

Foucault, M. (1990b): Tecnologías del yo. Buenos Aires: Siglo XXI.

Foucault, M. (1989): Vigilar y castigar. Buenos Aires: Siglo XXI.

Giddens, A. (1993): Consecuencias de la modernidad, Madrid, Alianza.

Gille, B. (1999): Introducción a la historia de las técnicas, Barcelona, Crítica.

Graubard, S. (comp.) (1999): El nuevo debate sobre la inteligencia artificial, Barcelona, Gedisa.

Gubern, R. (1996): Del bisonte a la realidad virtual, Barcelona, Anagrama.

Habermas, J. (1986): Ciencia y técnica como ideología, Madrid, Tecnos.

Han, B. (2015): El aroma del tiempo, Barcelona, Herder.

Han, B. (2014a): En el enjambre, Barcelona, Herder.

Han, B. (2014b): Psicopolitica. Neoliberalismo y nuevas técnicas de poder, Barcelona, Herder.

Heidegger, M. (1997): Filosofía, ciencia y técnica, Santiago de Chile, Editorial Universitaria. Hobsbawm, E. (2010): La era del capital, 1848-1875, Buenos Aires, Crítica.

Innis, H. (1950). Empire and communications. Oxford: Clarendon Press.

Jenkins, H. (2008): Convergence culture, Barcelona, Paidós.

Kittler, F. (1995): Aufschreibesysteme 1800 - 1900, München, Fink Verlag.

Kittler, F. (2014): Die Wahrheit der technischen Welt, Berlin, Suhrkmap. 
Kittler, F. (1999): Gramophone, film, typewriter, California, Stanford University Press.

Kyrou, A. (2010): Google God, Big Brother ne'existe pas, il est partout, Paris, Inculte.

Levy, P. (2007): Cibercultura: la cultura de la sociedad digital, México, Anthropos.

Lipovetsky, G. (2009): La pantalla global, Barcelona, Anagrama.

Manovich, L. (2006: El lenguaje de los nuevos medios de comunicación, Buenos Aires, Paidós.

Martin-Barbero, J. (1987): De los medios a las mediaciones, Barcelona, Gili.

Martinez Ojeda, B. (2006): Homo digitalis, Bogotá, Universidad de los Andes.

Marx, K. (1973): El capital, México, Fondo de Cultura Económica.

Mazlish, B. (1995): La cuarta discontinuidad, Madrid: Alianza.

McLuhan, M. (1996): Comprender los medios de comunicación, Buenos Aires, Paidós.

McLuhan, M. (1997): El medio es el mensaje, Buenos Aires, Paidós.

McLuhan, M. (1993): La aldea global, Barcelona, Gedisa.

Mc Hugh, G. (2011): Postinternet, Brescia, Link Editions.

Merleau-Ponty, M. (1993): Fenomenología de la percepción, Barcelona, Planeta.

Mitchell, W. (1995): City of bits: space, place and the infobahn, Massachusetts, MIT Press.

Mitchell, W. (1991): E-topía, Barcelona, Editorial Gustavo Gili.

Revel, J. (2009): Diccionaro Foucault, Buenos Aires, Nueva Visión.

Rifkin, J. (2014): La sociedad de coste marginal cero: el internet de las cosas, los bienes comunes y el eclipse del capitalismo, Barcelona, Paidós ibérica.

Rifkin, J. (2011): La tercera revolución industrial, Barcelona, Paidós.

Rosa, H. (2016): Alienación y aceleración. Hacia una teoría crítica de la temporalidad en la modernidad tardia, Buenos Aires, Katz Editores.

Sadin, E. (2017): La humanidad aumentada, la administración digital de mundo, Buenos Aires, Caja Negra.

Sadin, E. (2018): La silicolonización del mundo. La irresistible expansión del liberalismo digital, Buenos Aires: Caja Negra.

Sassen, S. (2000): Territorio, autoridad, derecho, Madrid, Katz.

Satoshi, Nakamoto (2008): Bitcoin A Peer-to-Peer Electronic Cash System, https://bitcoin. org/bitcoin.pdf.

Simondon, G. (2007): El modo de existencia de los objetos técnicos, Buenos Aires, Prometeo.

Stiegler, B. (2002): La técnica y el tiempo. Tomos I, II y III, Hondarribia, Hiru. (tres volúmenes)

Virilio, P. $\left(2003^{\mathrm{a}}\right)$ : Amanecer crepuscular, Buenos Aires: Fondo de Cultura Econoómica

Virilio, P. (2006): Ciudad pánico. El afuera comienza aquí, Buenos Aires, Libros del Zorzal.

Virilio, P. (2003b): El arte del motor. Aceleración y realidad virtual, Buenos Aires, Manantial.

Virilio, P. (1997ª): El cibermundo, la política de lo peor, Madrid, Cátedra.

Virilio, P. (1988): Estética de la desaparición, Barcelona, Anagrama.

Virilio, P. (1989): La máquina de visión, Madrid, Cátedra

Virilio, P. (1997b): La velocidad de liberación, Buenos Aires, Manantial

Virilio, P. (2005): The information bomb, London, Verso.

Virilio, P. (2012): The great accelerator, Cambridge, Polity Press.

Virilio, P. (2006): Velocidad y política, Buenos Aires, La marca editora.

Wajcman, J. (2017): Esclavos del tiempo, Paidós, Barcelona.

Weber, M. (2010): La ética protestante y el espíritu del capitalismo, Buenos Aires, Prometeo.

Wiener, N. (1988): Cibernética y sociedad, Buenos Aires, Sudamericana. 